\title{
Cutaneous T-cell Lymphoma
}

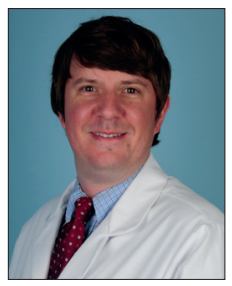

Paul L Haun MD MSc

Assistant Professor of Dermatology and Dermatopathology

University of Pennsylvania

Philadelphia, PA, USA

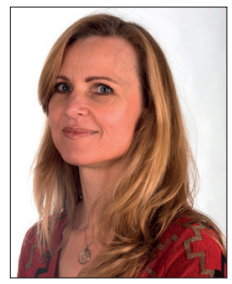

\section{Julia J Scarisbrick MBChB FRCP MD}

Honorary Professor, University Hospital Birmingham

and Division of Immunology \& Immunotherapy

University of Birmingham, UK

\section{Declaration of Independence}

This book is as balanced and as practical as we can make it.

Ideas for improvement are always welcome: fastfacts@karger.com

\section{KARGER}


Fast Facts: Cutaneous T-cell Lymphoma

First published 2019

Text @ 2019 Paul L Haun, Julia J Scarisbrick

(C) 2019 in this edition S. Karger Publishers Limited

S. Karger Publishers Limited, Elizabeth House, Queen Street, Abingdon, Oxford OX14 3LN, UK

Tel: +44 (0)1235 523233

Book orders can be placed by telephone (+41 61306 1440), email (orders@karger.com), or via the website at: karger.com

Fast Facts is a trademark of S. Karger Publishers Limited.

All rights reserved. No part of this publication may be reproduced, stored in a retrieval system, or transmitted in any form or by any means, electronic, mechanical, photocopying, recording or otherwise, without the express permission of the publisher.

The rights of Paul L Haun and Julia J Scarisbrick to be identified as the authors of this work have been asserted in accordance with the Copyright, Designs \& Patents Act 1988 Sections 77 and 78.

The publisher and the authors have made every effort to ensure the accuracy of this book, but cannot accept responsibility for any errors or omissions.

For all drugs, please consult the product labeling approved in your country for prescribing information.

Registered names, trademarks, etc. used in this book, even when not marked as such, are not to be considered unprotected by law.

A CIP record for this title is available from the British Library.

ISBN 978-1-912776-30-6

Haun PL (Paul)

Fast Facts: Cutaneous T-cell Lymphoma/

Paul L Haun, Julia J Scarisbrick

Medical illustrations by Annamaria Dutto, Withernsea, UK.

Typesetting by Thomas Bohm, User Design, Illustration and Typesetting, UK.

Printed in the UK with Xpedient Print.

Made possible by a contribution from Helsinn Healthcare S.A. Helsinn did not have any influence on the content and all items were subject to independent peer and editorial review. 
Introduction

5

Classification, epidemiology and etiology

Clinical presentation and differential diagnosis

Pathology and diagnosis of mycosis fungoides and Sézary syndrome

Pathology and diagnosis of non-mycosis fungoides CTCL

Staging and prognosis

Treatment

87

Useful resources

106

Index

108 


\section{List of abbreviations}

AITL: angioimmunoblastic T-cell lymphoma

ALK: anaplastic lymphoma kinase

ATLL: adult T-cell leukemia/ lymphoma

BPDCN: blastic plasmacytoid dendritic cell neoplasm

CAILS: Composite Assessment of Index Lesion Severity

CBC: complete blood count

CCR: chemokine (C-C motif) receptor

CD: cluster of differentiation

CLA: cutaneous lymphocyte antigen

CT: computed tomography

CTCL: cutaneous T-cell lymphoma

EBV: Epstein-Barr virus

ECP: extracorporeal photopheresis

EMA: epithelial membrane antigen

FDA: Food and Drug Administration

FMF: folliculotropic mycosis

fungoides

HDAC: histone deacetylase

HIV: human immunodeficiency virus

HTLV-1: human T-cell lymphotropic virus type 1

IFN: interferon

Ig: immunoglobulin

IL: interleukin

LDH: lactate dehydrogenase
LyP: lymphomatoid papulosis

MF: mycosis fungoides

MOP: methoxypsoralen

MRI: magnetic resonance imaging

nbUVB: narrow-band ultraviolet B

NGS/HTS: next generation sequencing/high throughput sequencing

NK: natural killer (cell)

PC-ALCL: primary cutaneous anaplastic large cell lymphoma

PET-CT: positron emission tomography-computed tomography

PUVA: psoralen plus ultraviolet light A therapy

SDT: skin-directed therapy

SPTCL: subcutaneous panniculitislike T-cell lymphoma

SS: Sézary syndrome

TCR PCR: T-cell receptor polymerase chain reaction

TGF: tumor growth factor

Th cell: T-helper cell

TIA-1: T-cell intracellular antigen

TSEBT: total skin electron beam therapy

UV: ultraviolet

WHO/EORTC: World Health Organization/European Organisation for Research and Treatment of Cancer 


\section{Introduction}

Although many cutaneous T-cell lymphomas (CTCLs) run a generally indolent clinical course, prompt and accurate diagnosis should be the rule, not the exception. In today's changing environment of medical care, patients may have to wait to see a dermatologist, even one without expertise in CTCL; the challenge of swift and accurate diagnosis therefore often lies with primary care providers and pathologists.

Clinicopathological correlation requires the correct history, description of lesions and clinical work-up. We have written this concise text, to specifically help primary care providers, pathologists and general dermatologists recognize some of the clinical presentations, histopathological patterns and work-up needed for the diagnosis of CTCL.

The clinical presentation of CTCLs can vary greatly (many consider it one of the 'great mimickers') and the histopathology and ancillary molecular studies performed to assist in the diagnosis of CTCL can be difficult to recognize, analyze and interpret. Furthermore, the clinical behavior and treatment responses of primary cutaneous lymphomas are frequently different from their nodal counterparts, making accurate diagnosis essential for management. Recognition of an atypical rash by the astute practitioner can lead to earlier biopsies. In turn, if given a strong clinical history, the pathologist can make informed decisions with respect to the histopathology findings under the microscope. This teamwork is the cornerstone of the robust clinicopathological correlation that is crucial to the diagnosis of CTCL.

All patients with mycosis fungoides, with the exception of those with the earliest stages of disease, should be referred to a dermatologist with a specialist interest in skin lymphoma. Those with early-stage CTCL that fails to respond to skin-directed therapy should also be referred. In the treatment chapter, we provide an overview of the management of mycosis fungoides/Sézary syndrome, explaining treatment options and therapeutic values. A wide range of options is available, although none is curative (other than, potentially, stem cell transplantation). Patients typically cycle through treatments, with 
periods of expectant therapy during which they receive palliation and symptomatic relief. Effective management requires knowledge of the wide range of options, how they are used, and their side-effect profile, in order to tailor treatment to the individual patient. Some of these treatments are advanced and must be managed by expert providers. Here, we offer basic information to make generalists aware of these options.

This book is a succinct basis for the understanding, diagnosis and work-up of CTCL by non-specialists, with the aim of improving collaboration between general clinicians and pathologists, while ensuring a greater awareness of how these conditions are managed. 